\title{
The Craftsperson, Tacit Knowledge, and Digital Embodiment
}

\author{
Carinna Parraman \\ Centre for Fine Print Research \\ University of the West of England, \\ Bristol, United Kingdom \\ Carinna.Parraman@uwe.ac.uk
}

\author{
Fabio D'Agnano \\ Centre for Fine Print Research \\ University of the West of England, \\ Bristol, United Kingdom \\ Fabio.DAgnano@uwe.ac.uk
}

\author{
Wuon-Gean Ho \\ Centre for Fine Print Research \\ University of the West of England, \\ Bristol, United Kingdom \\ Wuon-Gean.Ho@uwe.ac.uk
}

\section{INTRODUCTION}

We are increasingly living in a world of digital interfaces: touchscreens in our pockets and technology at home. Computing, robotics and $\mathrm{Al}$ are beginning to establish alternative approaches to creative expression. However, these machines still fall short at complex and fine motor tasks. Can they perform a surgical manoeuvre, throw a pot, weave a garment, or check the safety of food? Of course, machines have automation, scalability and tirelessness on their side, but the creative input is still up to the artist as author.

\subsection{Crafts skills}

Over the last century, craft has evolved as way of making things. Sennett draws a compelling picture of craftsmanship as an attitude to material culture spanning a range of professions from lab technicians to goldsmiths. "Craftsmanship names an enduring, basic human impulse, the desire to do a job well for its own sake" (Sennett 2008, p.9). Craft is an important bridge between art, design and industrial design; indeed it is the foundation for today's high-quality construction, printing and fabrication methods.

\subsection{Tacit knowledge}

Many traditional crafts skills involve the development of tacit knowledge, which is usually best gained through direct learning. Commonly, apprentices begin with observation, continue with repetition, develop autonomy, and finally achieve mastery. Tacit knowledge can be seen as an internal reference library that trains senses, builds muscular coordination and a complex understanding of materials.

\section{EUREKA MOMENTS}

Paquette (2018) talks about how some learners progress much faster than others in developing tacit knowledge because of sudden comprehension of threshold concepts, or eureka moments. The following section explores the things that may prompt tacit knowledge to develop in this way. For example, when we look at a material, we engage different perceptions to evaluate it (sight, touch, sound, smell, pressure, feel). Batcho et al (2016) concur: "When subjects learn a novel motor task, several sources of feedback (proprioceptive, visual or auditory) contribute to the performance."

\subsection{Constructed sound}

Complex tasks (defined as something which cannot be mastered in a single session) often rely on multimodal approach to learning (Sigrist et al. 2013). Can amplifying the sound of the task help increase haptic understanding?

According to Sternberg's definition, tacit knowledge is necessarily personal and requires individual learners to realise what does not work as well as what doe (Hedlund et al. 2000). Learners are often challenged by watching experts in time-based media, as the apparently seamless display of skill does not allow for an understanding of the nuances of the procedure (Wood et al. 2009). 


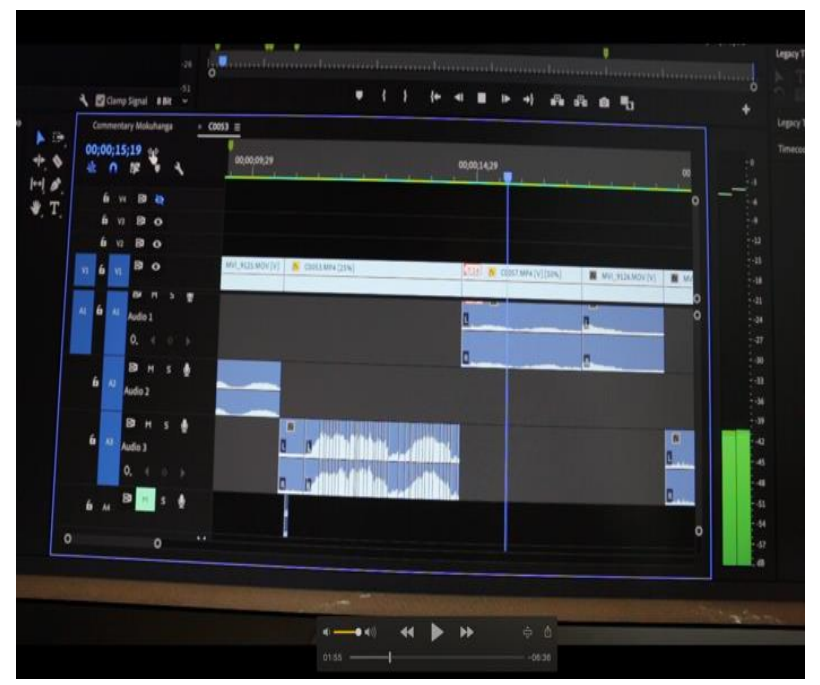

Figure 1: Still frame from "Three Observations" by Wuon-Gean Ho. Research Film (2020) at 1:56

Real-time and slow-motion footage was taken of a task which is usually performed in Japanese woodblock printing. The slow-motion footage has sound which is pitched too low for affectively natural comprehension. Real-time sound was cut, spliced and layered (Figure 1) in order to create a waveform approximation with longer duration to fit the slow-motion footage. The resulting combination of slower footage and naturally pitched sound allow learners to try to mimic the sounds heard. The aim is for a deeper understanding of tacit skills.

\subsection{Reproducing colour and texture}

How does a painter paint? How might one translate colour and texture as a sensory experience? Texture is a visual attribute that enables us to distinguish the differences between materials, identify the structure and shape of objects, and discriminate edges in a complex pictorial scene. One could consider texture as multisensory; first impressions are gleaned through sight, which may then be reinforced by smell, taste and touch (Klatzky \& Lederman 2010).

Inspired by the meticulous painting methods used by artists such as Van Gogh and Seurat, the objective was to create a vector-driven painting machine that applied a brush loaded with paint to paper in a methodical and mechanical way, yet remained human analogous. The difference was that although the vector marks (digital) were exactly repeatable, the painted brush strokes (analogue) were not. Based on the placement of the brush on the paper and the flow of paint, each painted brushstroke was similar but not exactly the same, thus creating a non-uniform but harmonious effect across the paper surface. The painting machine has inspired discussions about novel ways of applying paint that emulate the brush strokes of artists, demonstrating alternative methods of halftoning and colour separating, with the potential for tactile pictures for the visually impaired.

\subsection{Sculpture to 3D Relief}

Touch acts as a supplementary adjunct to sight, providing a lot of information about the real world, which is why it is of primary importance for people with visual impairment. Sight represents long-range touch with the sensation of colour and more; touch is a close view with less colour, supplemented with the sensation of roughness (Villey 1954).

The project UNESCO for All aimed to enable access to four UNESCO sites by translating art and architecture into tactile 3D models. Shape, colour and material differences were conveyed by texture. We used audio technology, enabling the blind and visually impaired to experience cultural heritage integrating tactile exploration with audio data.

Several tools were used to digitise the originals including photogrammetry, 3D modelling and digital sculpting. The models were made with a combination of CNC routing, laser cutting and engraving, SLS resin, and 3D printing (Figure 2). We combined techniques to overcome specific limitations of the tools and media in a quest to obtain precise replicas at a reasonable price and of considerable size.

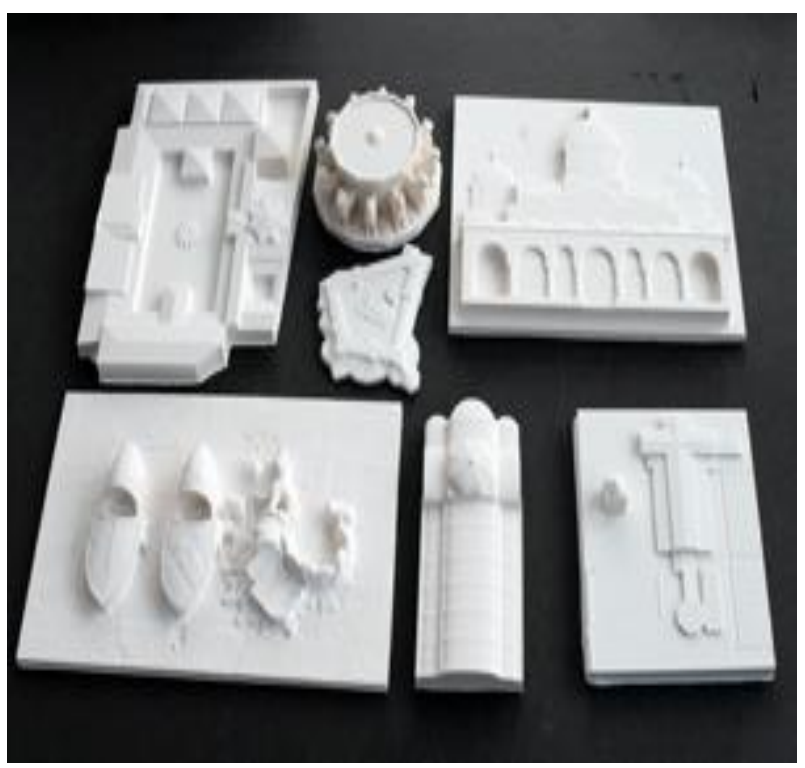

Figure 2: The tactile panels of the UNESCO for All project

\section{ACKNOWLEDGEMENTS:}

Project funded by UKRI, Research England, Expanding Excellence in England (E3) Fund. 


\section{REFERENCES}

Batcho, C.S., Gagné, M., Bouyer, L.J., Roy, J.S. and Mercier, C. (2016) Impact of online visual feedback on motor acquisition and retention when learning to reach in a force field. Neuroscience. 337, pp. 267-275.

Hedlund J., Antonakis J., Sternberg R.J. (2000) Tacit Knowledge and Practical Intelligence: Understanding the Lessons of Experience, Yale University Press.

Klatzky R. L., and Lederman, S. J. (2010) Multisensory Texture Perception. In: Naumer M and Kaiser, J. Multisensory Object Perception in the Primate Brain, NY, Springer, pp. 211-230.
Paquette, A (2018) Spatial Visualization and Professional Competence: The Development of Proficiency Among Digital Artists, Springer.

Sigrist, R., Rauter, G., Riener, R. et al. (2013) Augmented visual, auditory, haptic, and multimodal feedback in motor learning: A review. Psychonomic Bulletin Review, 20, pp. 21-53

Sennett, R (2008) The Craftsman, Yale University Press.

Wood, N. et al. (2009) A tacit understanding: The designer's role in capturing and passing on the skilled knowledge of master craftsmen. International Journal of Design, 3 (3), pp. 65-78.

Villey, P. (1954) Le Monde des aveugles, Librairie Jose Corti 